\title{
Modelagem Numérica dos Efeitos da RAA Conforme a Temperatura
}

\author{
Edmilson Lira. Madureira ${ }^{1 *}$ \\ *Autor de Contacto: edmadurei@yahoo.com.br \\ ${ }^{1}$ Departamento de Engenharia Civil, Centro de Tecnologia, Universidadade Federal do Rio Grande do Norte, Natal, \\ Brasil
}

\section{RESUMO}

A Reação Álcali-Agregado, RAA, foi reportada por Stanton em 1940, e, seus efeitos repercutiram a partir da década de oitenta, em razão de danos provocados em estruturas de concreto, caracterizados pela fissuração localizada, ruína global e comprometimento da funcionalidade. A despeito do volume considerável de pesquisas realizadas a solução do problema permanece incipiente. A simulação numérica constitui recurso pertinente à avaliação dos defeitos decorrentes da RAA e à consequente recuperação estrutural. $\mathrm{O}$ desenvolvimento, extensão e cronologia das avarias decorrentes da reação, são influenciados por uma diversidade de fatores, a exemplo da umidade, da taxa de armadura do elemento estrutural, das tensões confinantes e da temperatura. $\mathrm{O}$ objetivo deste trabalho é a análise da influência da temperatura sobre o desempenho mecânico de vigas, afetadas pelo efeito deletério associado à RAA. Para o seu cumprimento é empregado "software" desenvolvido com base no método dos elementos finitos, relações constitutivas não lineares em estado plano de tensões para o concreto, e, um modelo termodinâmico para a avaliação de suas deformações por RAA. Os resultados obtidos indicaram que o declínio da resistência inicial do concreto, resultou na diminuição da margem de segurança ao colapso, que em alguns casos foi deflagrado, e, para as temperaturas mais elevadas foi substancialmente antecipado.

Palavras chave: Simulação; Vigas; Temperatura; Reação Álcali-Agregado

\section{INTRODUÇÃO}


A Reação Álcali-Agregado, RAA, transformação química envolvendo os álcalis do cimento e minerais dos agregados do concreto, foi reportada por Stanton em 1940 e despertou interesse no âmbito da Engenharia Civil a partir da década de oitenta, devido a danos estruturais decorrentes de seus efeitos então constatados. As deficiências decorrentes incluíam fissuração local, ruína generalizada e comprometimento da funcionalidade.

Apesar do volume considerável de pesquisas a efetiva solução do problema ainda requer o aperfeiçoamento da teoria envolvendo a descrição do fenômeno experimentalmente fundamentado. A simulação numérica constitui recurso fundamental para a estimativa das avarias provocadas pela RAA, podendo auxiliar a tomada de decisões quanto à recuperação estrutural, e, servir de base para modificações de critérios de dimensionamento.

O desenvolvimento, extensão e cronologia de danos do efeito expansivo da RAA são influenciados por extensa diversidade de fatores condicionantes, tais como o teor de umidade, a taxa de armadura do membro estrutural, as tensões confinantes e a temperatura.

$O$ objetivo deste trabalho é a simulação numérica do desempenho mecânico de vigas de concreto armado, afetado pela ação deletéria da Reação Álcali-Agregado.

Com vistas ao atendimento de tal finalidade foi empregado "software" desenvolvido com base no método dos elementos finitos e relações constitutivas não lineares, e, um modelo termodinâmico de materiais porosos reativos, para a avaliação das deformações do concreto por RAA.

A análise foi realizada em estado plano de tensões sobre modelos diferenciados entre si pela taxa de armadura, pela intensidade do carregamento e pela temperatura.

\section{MODELAGEM}

Em problemas bidimensionais, a matriz constitutiva referente ao modelo ortotrópico não linear pode ser definida com base nas deformações equivalentes dadas por:

$$
\varepsilon_{e i}=\varepsilon_{i}+D_{i j} \varepsilon_{j} / D_{i i}
$$

Os índices “i $\mathbf{i}$ ” e “j” representam as direções principais, os “ $\mathbf{D}_{\mathbf{i j}}$ " os elementos da matriz constitutiva, e os parâmetros $\boldsymbol{\varepsilon}_{\mathbf{i}}$ e $\boldsymbol{\varepsilon}_{\mathbf{j}}$ são as deformações segundo as direções principais.

Para o concreto em compressão foram adotadas as relações de (Hognestad, 1951):

$$
\begin{gathered}
\sigma=\frac{2 . \sigma_{p}}{\varepsilon_{p}}\left(1-\frac{\varepsilon}{2 . \varepsilon_{p}}\right) \cdot \varepsilon \quad \text { para } \varepsilon_{\mathrm{p}}<\varepsilon<0 \\
\sigma=\sigma_{p}\left(1-\frac{3}{20} \frac{\varepsilon-\varepsilon_{p}}{\varepsilon_{c u}-\varepsilon_{p}}\right) \text { para } \varepsilon_{\mathrm{cu}}<\varepsilon<\varepsilon_{\mathrm{p}}
\end{gathered}
$$

onde " $\sigma_{\mathbf{p}}$ " é a tensão de pico do concreto, " $\boldsymbol{\varepsilon}_{\mathbf{p}}$ " a deformação a ela correspondente, e, " $\boldsymbol{\varepsilon}_{\mathbf{c u}}$ " a deformação limite última de ruptura do material. Essas equações representam os trechos de endurecimento e de amolecimento, seguimentos $\mathbf{O A}$ e $\mathbf{A B}$, respectivamente, da curva tensãodeformação para o concreto, figura 1.a.

Para o concreto em tração, trecho OCD da curva da figura 1.a, adotou-se um modelo de fissuras distribuídas, cujas vantagens são considerar a continuidade do campo de deslocamentos, e, dispensar modificações topológicas da malha de elementos finitos, no decorrer da análise. 
As tensões limite são definidas a partir da envoltória de (Kupfer and Gerstle, 1973), figura 1.b. O comportamento do aço, por sua vez, foi considerado elástico perfeitamente plástico.

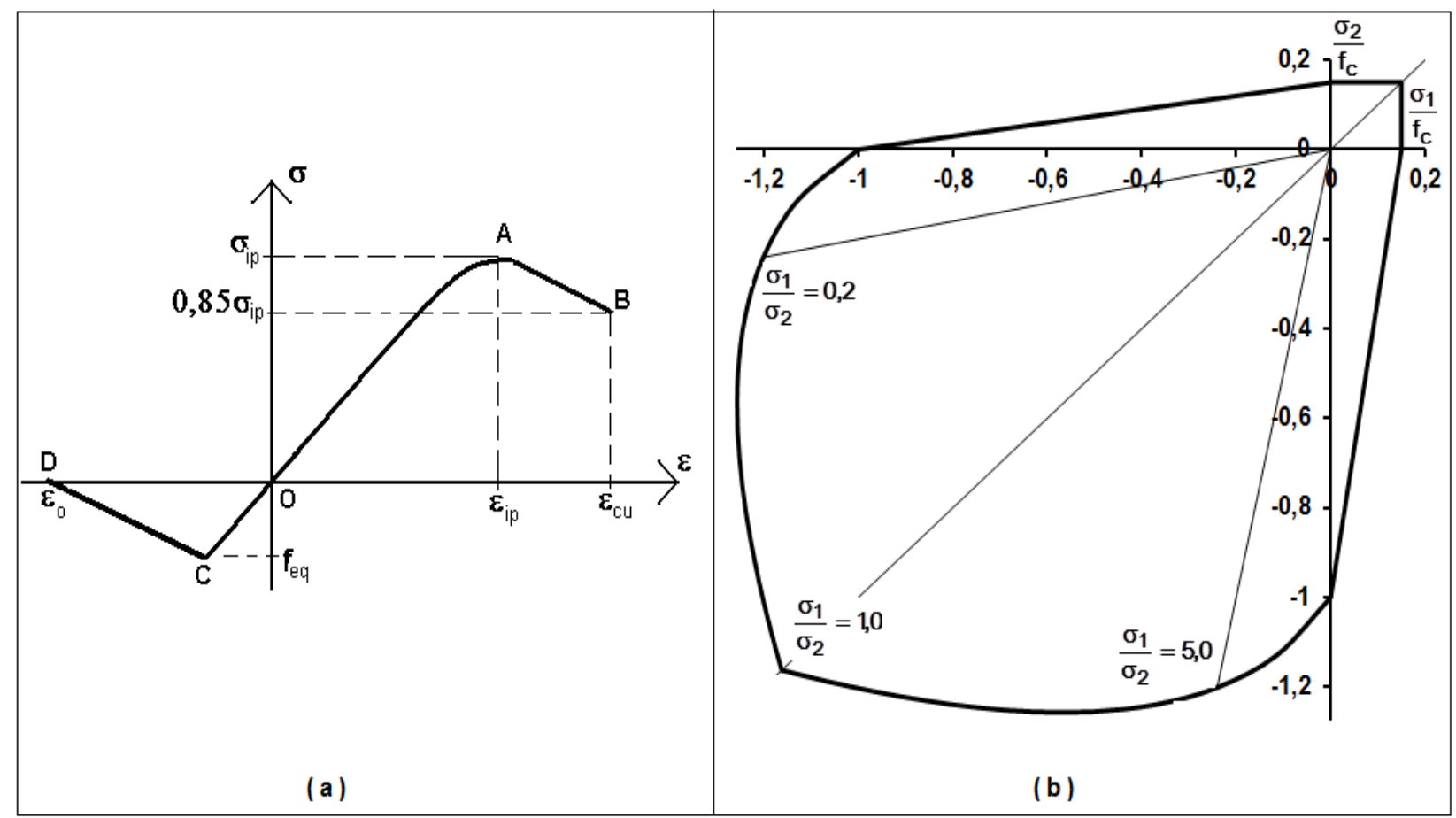

Figura 1. a ) Curva tensão deformação para o concreto; b ) Envoltória de ruptura do concreto

Para a simulação das expansões com o tempo, foi adotada formulação termodinâmica, assim denominada porque correlaciona as deformações por RAA com a cinética da reação química. (Capra and Sellier, 2002) propuseram para modelo termodinâmico a formulação:

$$
\begin{aligned}
& \varepsilon^{R A A} \approx 0 \text { para } \mathrm{A}<\mathrm{A}_{0} \\
& \varepsilon^{R A A}=\frac{\varepsilon_{o}}{A_{o}}\left(A-A_{o}\right) \text { para } \mathrm{A}>\mathrm{A}_{0}
\end{aligned}
$$

onde "A" é o teor de álcalis consumido na reação; "A," é o valor de "A" ao início da expansão do concreto, e, " $\varepsilon_{o}$ " é a parcela a ser deduzida da deformação, para considerar a defasagem entre os inícios da reação e da expansão da matriz de concreto, figura 2.

Para a modelagem da cinética da reação (Capra and Bournazel, 1998), propuseram a equação:

$$
\frac{d A}{d t}=k(T)(1-A)
$$

para a qual "k(T)" é a constante cinética associada à lei de Arhenius, avaliada a partir de:

$$
k(T)=k_{o} e^{\frac{-E_{a}}{R T}}
$$

onde "E $\mathbf{a}$ " é a energia de ativação, "R" a constante do gás ideal, "T' a temperatura, e, "ko" a constante cinética. A solução da equação 4 deve ser: 


$$
A=1-\left(1-A_{o}\right) e^{-k t}
$$

Resultando, para a equação 3 no intervalo $\mathbf{A}>\mathbf{A}_{\mathbf{0}}$, a forma:

$$
\varepsilon^{R A A}(t, T)=\frac{\varepsilon_{o}}{A_{o}}\left(1-A_{o}\right)\left(1-e^{-\left(k_{o} e^{-E_{a / R T}}\right) t}\right)
$$

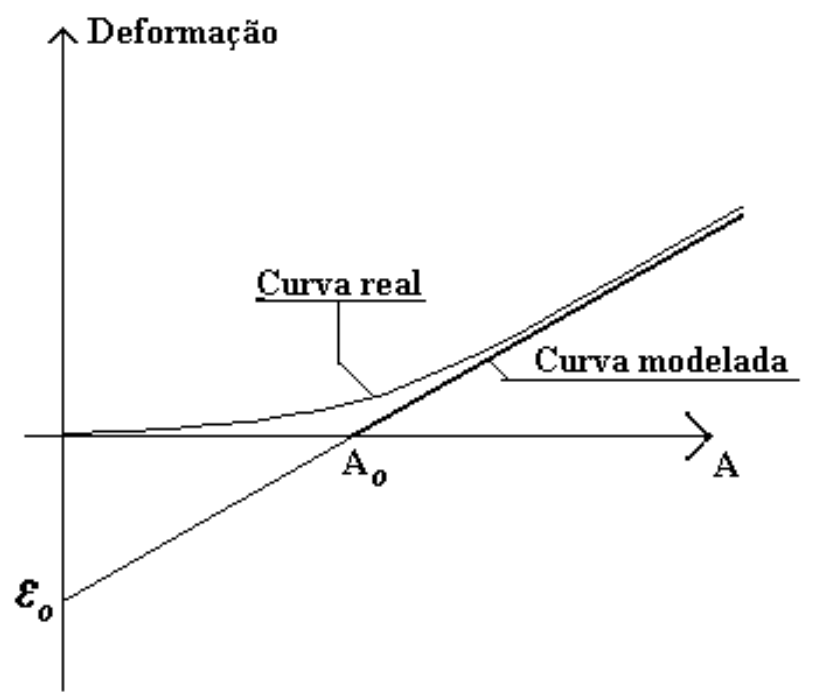

Figura 2. Esquema gráfico para definição dos parâmetros " $\varepsilon_{\mathrm{o}}$ " e " $\mathrm{A}_{\mathrm{o}}$

A influência das tensões confinantes foi considerada mediante o critério proposto por (Charlwood, 1994), expresso na forma:,

$$
\begin{gathered}
0 \leq \sigma_{i} \leq \sigma_{L} \rightarrow \varepsilon_{g}=\varepsilon_{u} \\
\sigma_{L} \leq \sigma_{i} \leq \sigma_{\max } \rightarrow \varepsilon_{g}=\varepsilon_{u}-K \cdot \log _{10}\left(\sigma_{i} / \sigma_{L}\right)
\end{gathered}
$$

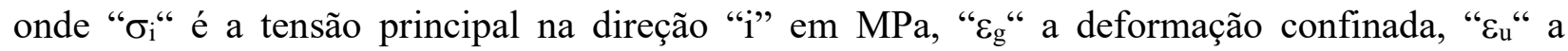
deformação não confinada. O parâmetro " $\sigma_{L}$ " é a tensão abaixo da qual a expansão é livre e deve ser avaliado em 0,30 MPa. O parâmetro " $\sigma_{\max }$ “ é a tensão para a qual a deformação por RAA é nula, cujo valor é da ordem de $8.0 \mathrm{MPa}$, e "K” é a Inclinação da reta $\varepsilon \times \log \sigma$.

A influência da umidade é expressa mediante a curva de (Poole, 1992):

$$
F(H)=H^{m}
$$

onde "m" é um parâmetro de ajuste, considerado igual a 8, e "H" é o teor de umidade.

A degradação do concreto por RAA foi modelada conforme (Pietruszczac, 1996), adaptada à taxa de variação das deformações por RAA do modelo de (Capra and Sellier, 2002), resultando: 


$$
E=E_{o}\left[1-\left(1-A_{E}\right)\left(1-\frac{1}{1-A_{o}} e^{-\left(k_{o} e^{-E_{a} / R T}\right) t}\right)\right]
$$

e,

$$
f_{c}=f_{c o}\left[1-\left(1-A_{f}\right)\left(1-\frac{1}{1-A_{o}} e^{-\left(k_{o} e^{-E_{a / R T}}\right) t}\right)\right]
$$

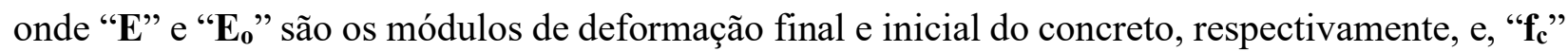

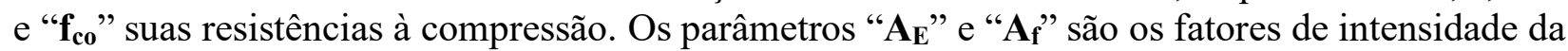
degradação do módulo de elasticidade e da resistência à compressão do concreto, respectivamente.

\section{SUPORTE COMPUTACIONAL}

Foi utilizado o "software" Análise Constitutiva Não Linear - ACNL, baseado no método dos elementos finitos (MEF) e escrito em linguagem FORTRAN. O referido código computacional inclui em sua pauta algorítmica a formulação dos elementos isoparamétricos de aproximação quadrática, lineares L3, figura 3.a, voltados para a simulação das barras de aço, e planos quadriláteros Q8, figura 3.b, destinados à discretização da região da massa de concreto.

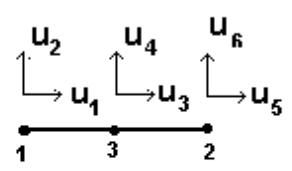

(a)

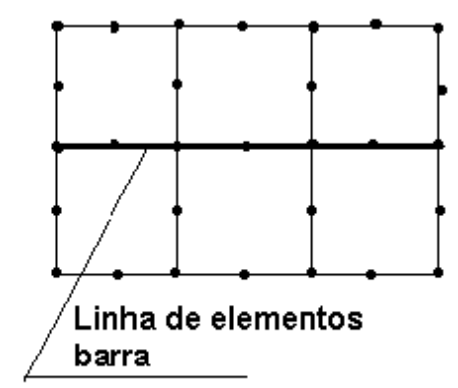

(b)

Figura 3. Elementos finitos: a - ) Lineares L3; b - ) Planos Q8

\section{VALIDAÇÃO DO SOFTWARE}

Um pilar de concreto de 3,00 m de comprimento e seção retangular com dimensões de $0,25 \mathrm{~m} \mathrm{x}$ 0,40 m, armado com quatro barras de $10 \mathrm{~mm}$ de diâmetro foi analisado em estado plano de tensões utilizando-se o programa, e, mediante modelagem simplificada em estado de solicitação uniaxial, pautada nos postulados da mecânica dos sólidos (Rodrigues, 2014), constatando-se boa concordância de resultados, tabela 1 .

Tabela 1. Deslocamentos por RAA

\begin{tabular}{|c|c|c|c|}
\hline Tempo (Dias) & \multicolumn{3}{|c|}{ Deslocamentos $\left.\mathbf{( 1 0}^{-3} \mathbf{~ m m}\right)$} \\
\hline & Modelo teórico & Modelo numérico & Diferença( \% ) \\
\hline 75 & 5,27 & 5,24 & 0,6 \\
\hline 150 & 9,90 & 9,80 & 1,0 \\
\hline 1200 & 28,30 & 28,10 & 0,7 \\
\hline 2500 & 41,20 & 40,90 & 0,7 \\
\hline
\end{tabular}




\section{MODELOS ANALISADOS}

Foram analisadas vigas com as dimensões ilustradas na figura 4, manufaturadas em concreto C20, com módulo de deformação e coeficiente de Poisson de $21300 \mathrm{MPa}$ e 0,17 , respectivamente, armado com barras de aço CA-50, distribuídas conforme figura 4.

A energia de ativação foi avaliada em $8500 \mathrm{~m}^{3} \cdot \mathrm{Pa} \cdot \mathrm{mol}^{-1}$, Ghanem et al,. (2010), e, para o número de Avogadro foi adotado o valor 6,02214129 x $10^{23} \mathrm{~mol}^{-1}$. Os fatores de intensidade da degradação do módulo de elasticidade, e, da resistência à compressão do concreto foram fixados em $\mathbf{A}_{\mathbf{E}}=0,3$ e $\mathbf{A}_{\mathbf{f}}=0,4$, respectivamente.

Os escoramentos das vigas são retirados aos 30 dias de idade do concreto quando foram carregadas mediante ação uniformemente distribuída ao longo de toda a sua extensão longitudinal, figura 4 . A data limite para observação do fenômeno foi fixada em 8000 dias.

Aproveitando a simetria do problema, o seu domínio no plano "xy" pôde ser definido conforme figura 4 que, uma vez discretizado adotando-se dimensão igual a $0,10 \mathrm{~m}$, resultou em malha de elementos finitos com 186 elementos planos e 62 elementos unidimensionais.

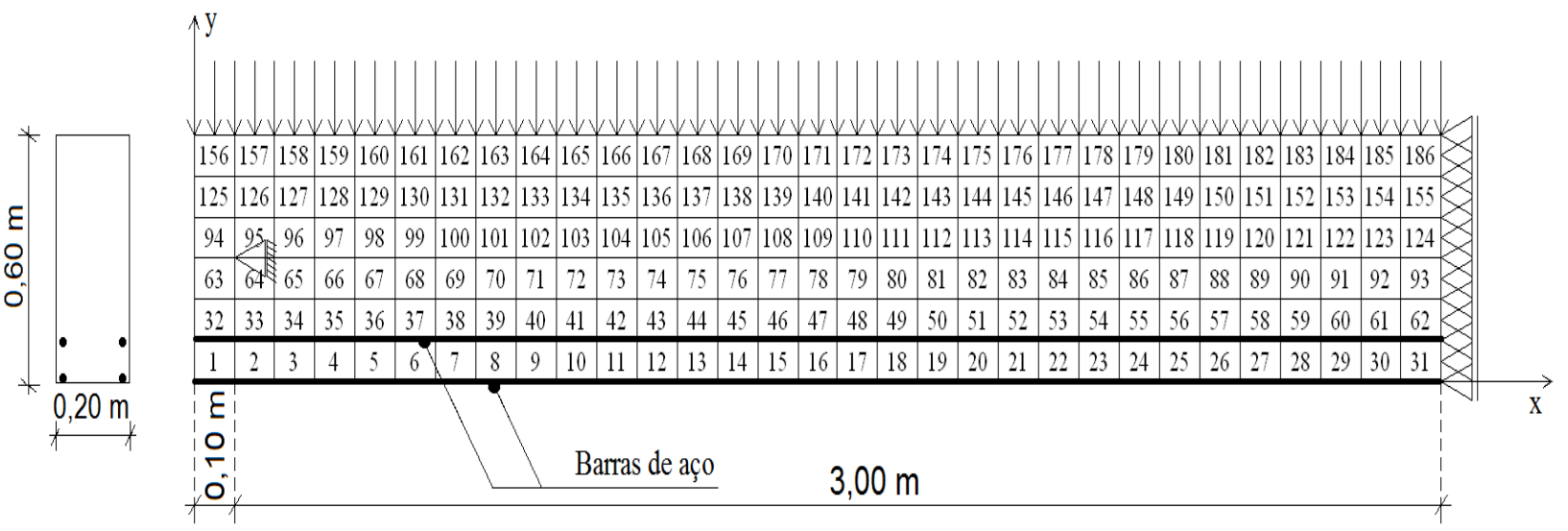

Figura 4. Domínio do problema e malha de elementos finitos

Tabela 2. Modelos estudados

\begin{tabular}{|c|c|c|c|c|c|c|c|}
\hline Caso & $\begin{array}{c}\mathbf{T} \\
\left({ }^{\circ} \mathbf{C}\right)\end{array}$ & As $\left(\mathrm{cm}^{2}\right)$ & $\sigma(\mathrm{MPa})$ & Caso & $\mathbf{T}\left({ }^{\circ} \mathbf{C}\right)$ & $\operatorname{As}\left(\mathrm{cm}^{2}\right)$ & $\sigma(\mathrm{MPa})$ \\
\hline 1 & 20 & 3,20 & 1 & 15 & 50 & 5,00 & 9 \\
\hline 2 & 20 & 3,20 & 5 & 16 & 50 & 8,00 & 1 \\
\hline 3 & 20 & 3,20 & 9 & 17 & 50 & 8,00 & 5 \\
\hline 4 & 20 & 5,00 & 1 & 18 & 50 & 8,00 & 9 \\
\hline 5 & 20 & 5,00 & 5 & 19 & 100 & 3,20 & 1 \\
\hline 6 & 20 & 5,00 & 1 & 20 & 100 & 3,20 & 5 \\
\hline 7 & 20 & 8,00 & 1 & 21 & 100 & 3,20 & 8 \\
\hline 8 & 20 & 8,00 & 5 & 22 & 100 & 5,00 & 1 \\
\hline 9 & 20 & 8,00 & 9 & 23 & 100 & 5,00 & 5 \\
\hline 10 & 50 & 3,20 & 1 & 24 & 100 & 5,00 & 9 \\
\hline 11 & 50 & 3,20 & 5 & 25 & 100 & 8,00 & 1 \\
\hline 12 & 50 & 3,20 & 9 & 26 & 100 & 8,00 & 5 \\
\hline 13 & 50 & 5,00 & 1 & 27 & 100 & 8,00 & 9 \\
\hline 14 & 50 & 5,00 & 5 & & & & \\
\hline
\end{tabular}


Foram analisados vinte e sete casos diferenciados entre si pela temperatura, pela taxa de armadura e pela intensidade estimada para a tensão solicitante, tabela 2.

A análise se refere a pontos da viga localizados nas proximidades do centro de seu vão.

Neste trabalho, a razão entre a tensão solicitante e a respectiva tensão resistente, aqui denominada Tensão Normalizada, foi adotada como parâmetro de referência indicativo da proximidade da ruína do concreto. A condição última referente à perda de capacidade do material em transmitir esforços é considerada iminente quando o valor de tal parâmetro se aproximar da unidade.

\section{RESULTADOS}

Para os casos "2", "5", “8”, "11", “14”, “17”, “20”, "23” e "26", que correspondem às vigas submetidas à tensão máxima de compressão no ponto de referência da ordem de $5 \mathrm{MPa}$, a configuração de equilíbrio para o carregamento solicitante pode ser representada, em termos de deslocamentos e tensões, pelos campos das Figuras 5 e 6. Constata-se, portanto, deslocamento vertical descendente no meio do vão do membro estrutural da ordem de 3,5 mm. Tal deslocamento foi de 7,0 mm, para os casos referentes à tensão de $9 \mathrm{MPa}$, e de $0,6 \mathrm{~mm}$, para o caso no qual a viga é isenta de carregamento externo suportando assim, exclusivamente, seu peso próprio, Tabela 3.
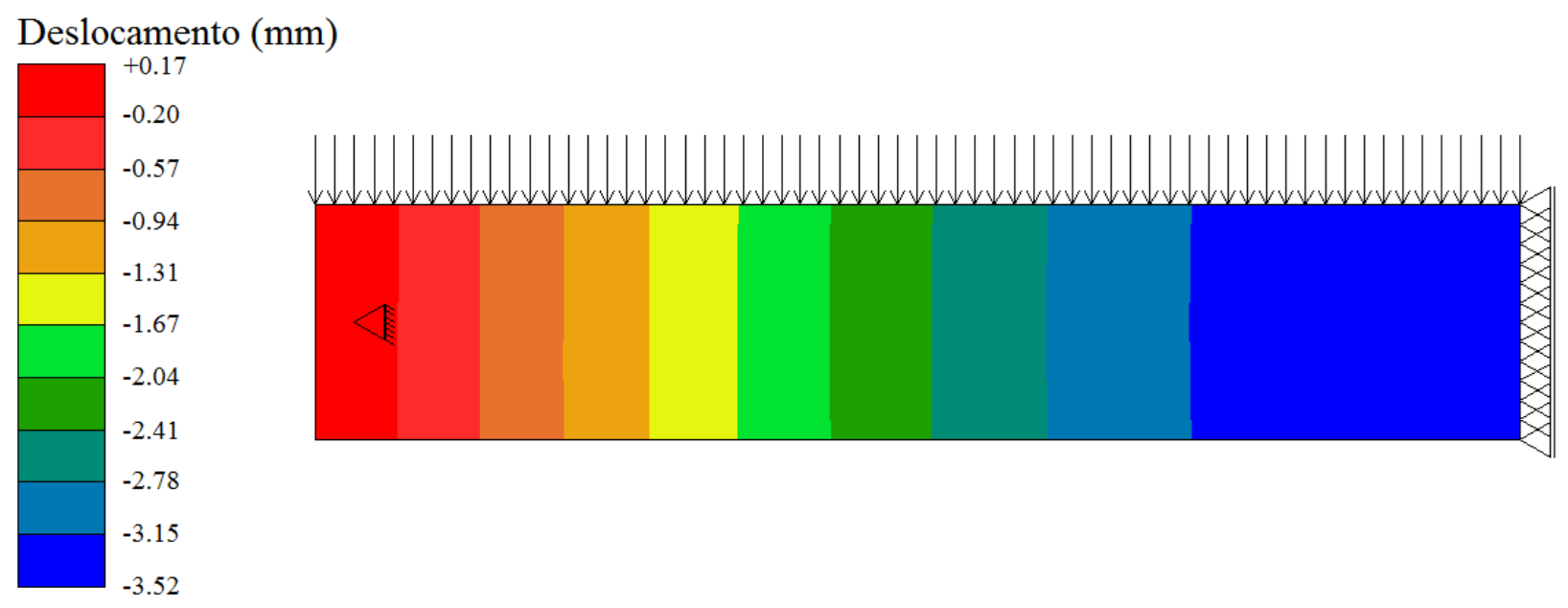

Figura 5. Deslocamento imediato ao carregamento para o caso "2"

Uma vez deflagrado o efeito expansivo da RAA na massa sólida de concreto, as vigas deformaramse progressivamente e os deslocamentos verticais do ponto de referência processaram-se em sentido ascendente, tendendo, portanto a reverter os deslocamentos imediatos. Tais movimentações evoluíram conforme as curvas da Figura 7 e estabilizaram, em geral, após 6000 dias, quando assumiram a distribuição do campo ilustrado na Figura 8.

Para os casos " 1 ", "4" e "10", referentes ao modelo na condição de viga descarregada, o acréscimo final de deslocamentos por RAA foi da ordem de 2,3 mm, Tabela 3, correspondendo a 3,6 vezes o valor constatado no instante imediato à remoção do escoramento do membro estrutural. Em se tratando dos casos " 2 ", "11" e "20", para os quais o modelo é submetido à tensão solicitante de 5 $\mathrm{MPa}$, os deslocamentos adicionais por RAA estabilizaram-se em torno de $1,8 \mathrm{~mm}$, valor que representa 50\% do deslocamento apresentado no instante imediato ao carregamento, Tabela 3. Para os casos " 3 ", "12" e "21" para os quais o modelo é solicitado mediante tensão da ordem de 8,0 $\mathrm{MPa}$, por sua vez, os deslocamentos adicionais por RAA, atingiram valor em torno de $1,1 \mathrm{~mm}$, e, portanto, com magnitudes inferiores a $15 \%$ do deslocamento imediato ao carregamento, Tabela 3. Para os demais casos estudados o desempenho da viga, em face do efeito expansivo decorrente da Reação álcali-Agregado, foi semelhante, diferindo, sobretudo, nos valores obtidos para o parâmetro 
em destaque, Tabela 3. Ressalta-se que, para tensões de compressão de maior intensidade, os deslocamentos por RAA assumiram magnitude menos significativas. Examinando-se ainda as curvas da Figura 7, constata-se que os deslocamentos por RAA são tanto menores, quanto maior a taxa da armadura de aço.

Tensão $(\mathrm{MPa})$

\begin{tabular}{|l|l|}
\hline & +1.08 \\
& +0.81 \\
& +0.54 \\
& +0.27 \\
& 0.00 \\
\hline & 0.00 \\
\hline & -0.62 \\
\hline & -1.24 \\
\hline & -1.86 \\
-2.48 \\
-3.10 \\
-3.72 \\
-4.34 \\
-4.96
\end{tabular}

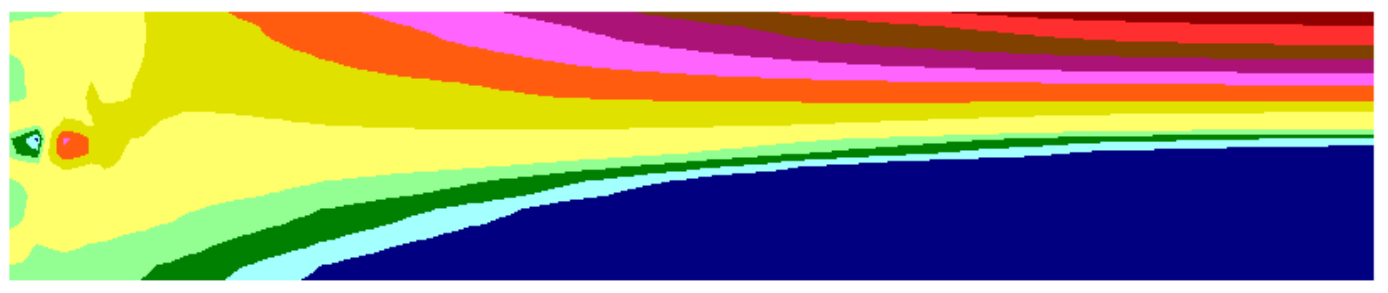

Figura 6. Tensões na direção "x" imediatas ao carregamento para o caso "2"

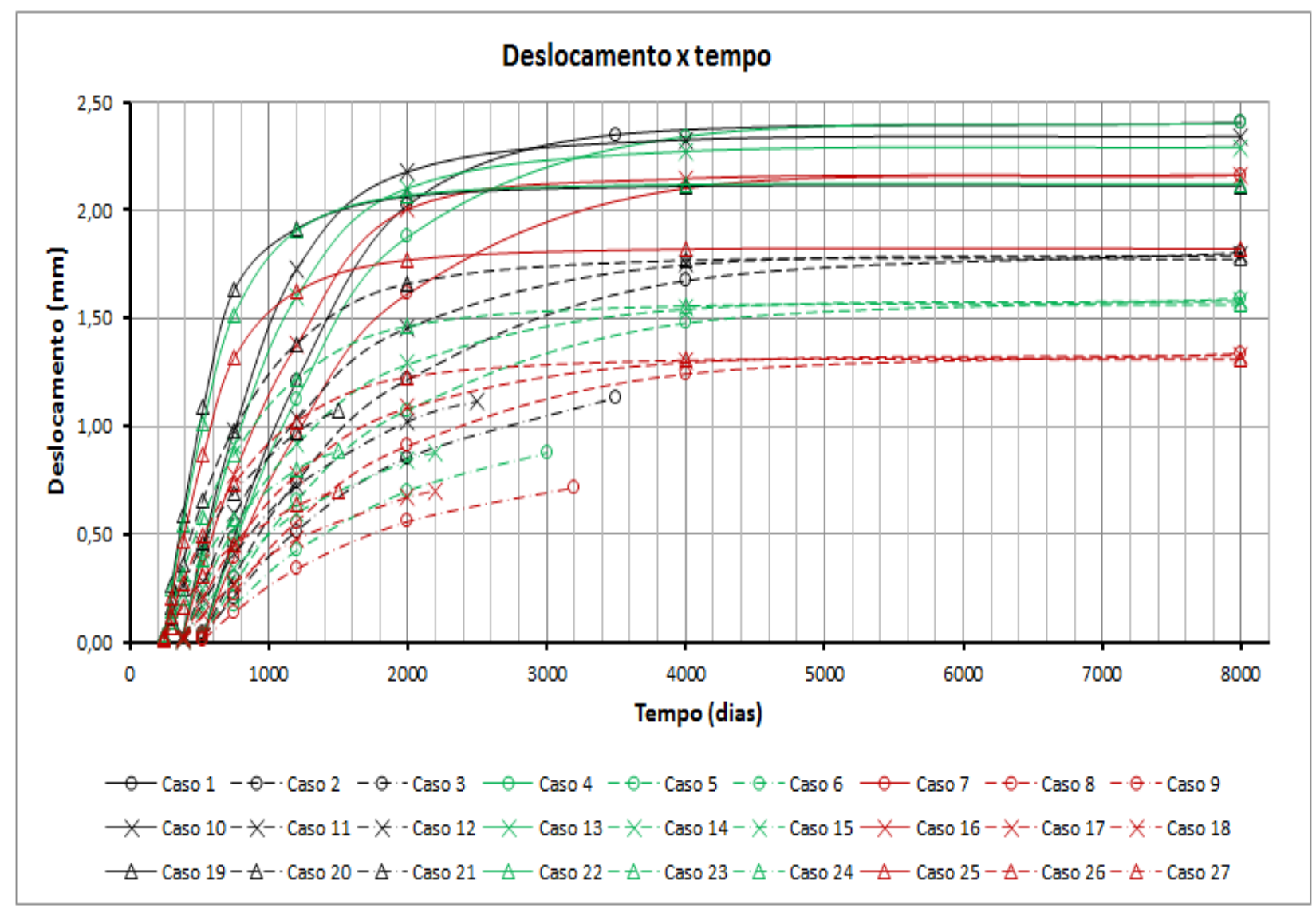

Figura 7. Deslocamentos na direção "x" devidas à RAA 
Deslocamento $(\mathrm{mm})$
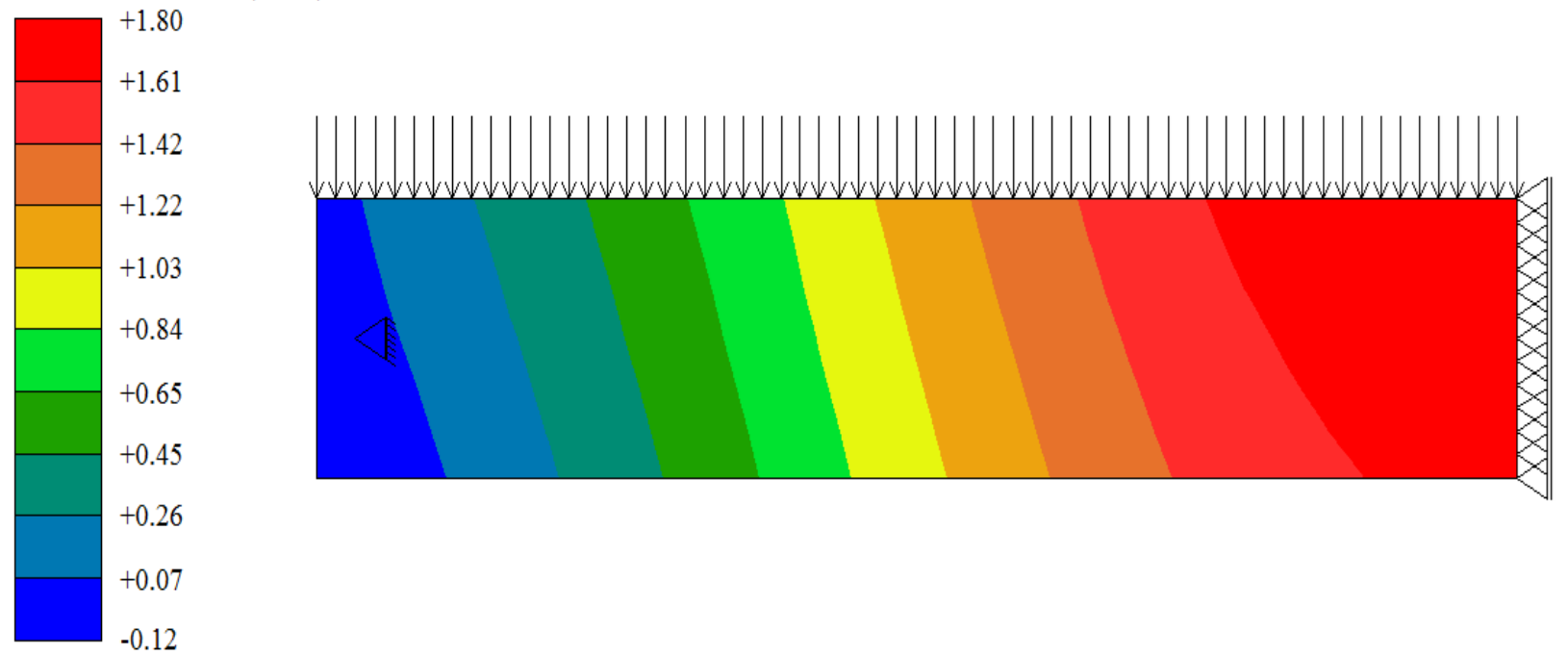

Figura 8. Deslocamento por RAA aos 8000 dias de idade para o caso "2"

Para os casos de "1" a "9", Figura 9, com temperatura fixada em $20^{\circ} \mathrm{C}$, os deslocamentos decorrentes da RAA só tiveram início aos 510 dias de idade do concreto, enquanto, para os casos de "10" a "18", referentes à temperatura de $50^{\circ} \mathrm{C}$, e para os casos de "19" a "27", concernentes à temperatura de $100^{\circ} \mathrm{C}$, o início dos deslocamentos se deu aos 370 dias e 240 dias, respectivamente, corroborando assim o efeito estimulante da temperatura sobre o fenômeno.

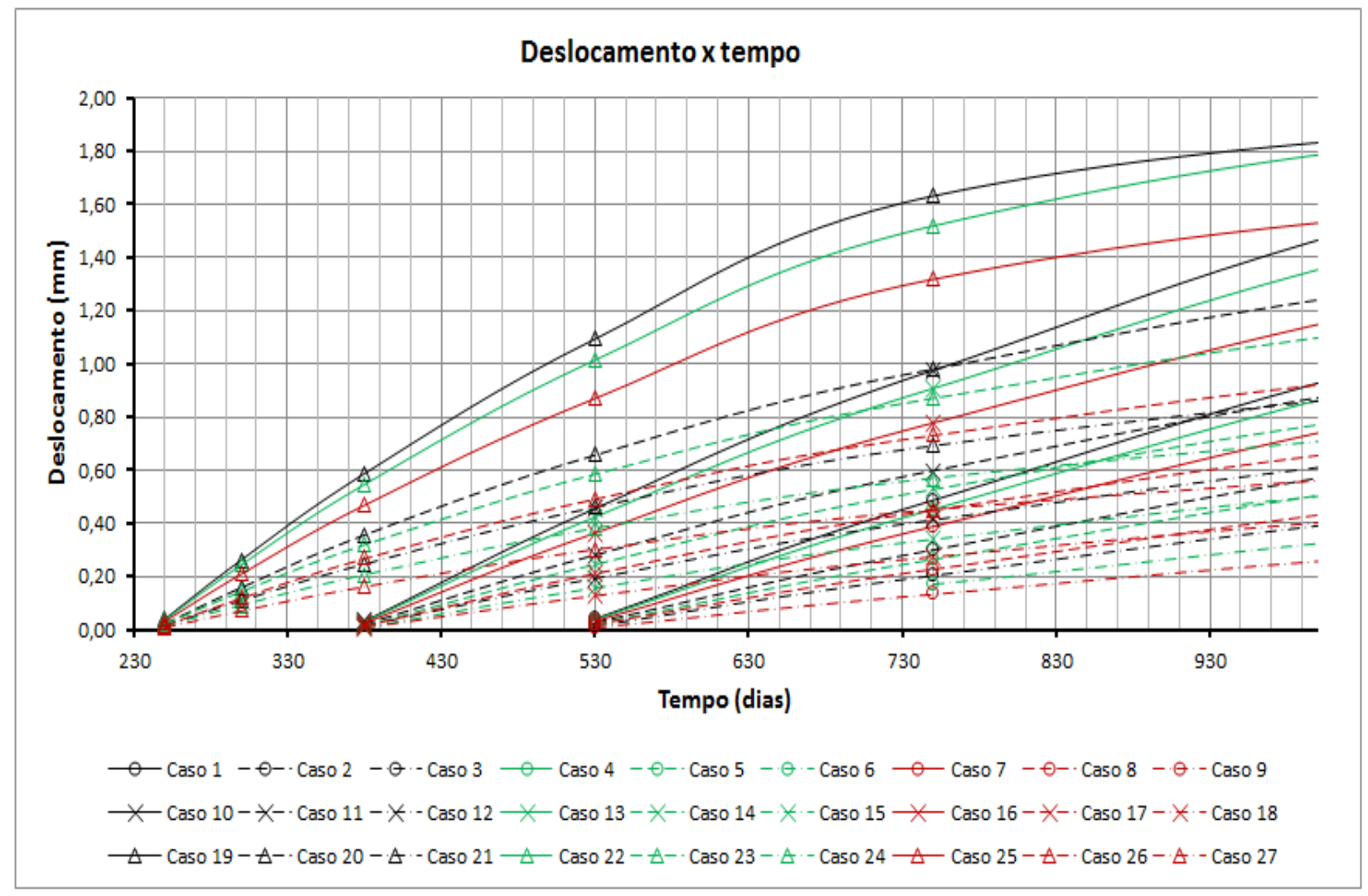

Figura 9. Início dos deslocamentos na direção "x" devidas à RAA 
Os resultados obtidos revelaram que, para os casos nos quais a viga é submetida a carregamento externo, as deformações por RAA promoveram o alívio de tensões de compressão no concreto com o tempo, na região central de seu banzo superior, embora em níveis bem discretos, Figura 10. O mesmo se dá para os casos em que a viga é solicitada exclusivamente pelo peso próprio, e são providas de seções de barras de aço com áreas de $3,20 \mathrm{~cm}^{2}$ e $5,00 \mathrm{~cm}^{2}$. Entretanto, na hipótese em que a área da seção transversal da armadura é de $8,00 \mathrm{~cm}^{2}$, verifica-se tendência contrária, o que pode ser explicado pela maior efetividade do efeito de tirante das barras de aço de maior área e, portanto, de maior rigidez.

A análise comparativa das Figuras 11 e 12, que se referem ao caso "6", além decorroborar o comportamento em termos gerais, relatado no parágrafo anterior, assinala uma ampliação perceptível da região comprimida da viga. Os demais casos apresentaram similar.

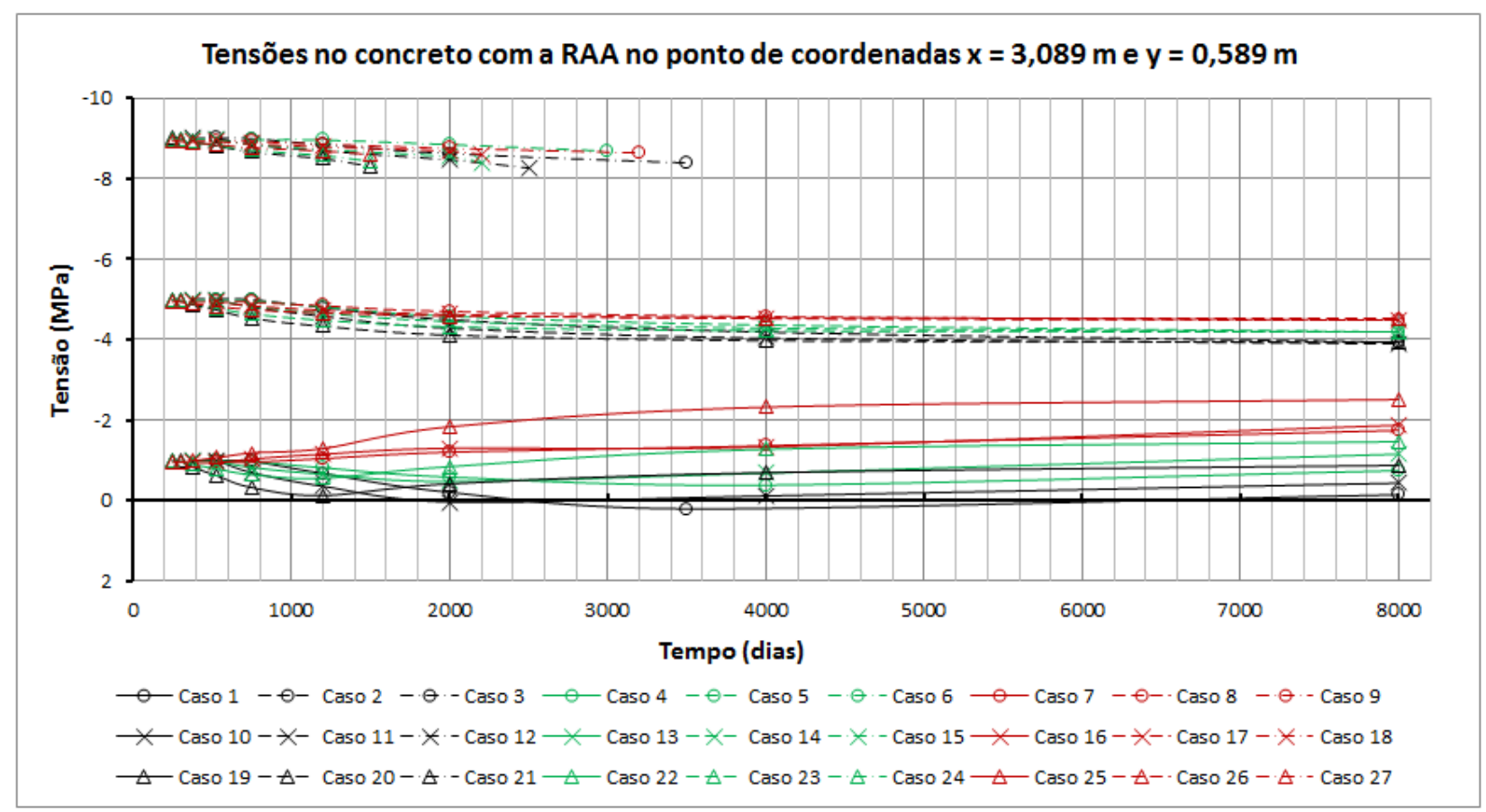

Figura 10. Tensões no concreto na direção "x" devidas à RAA

Tensão (MPa)

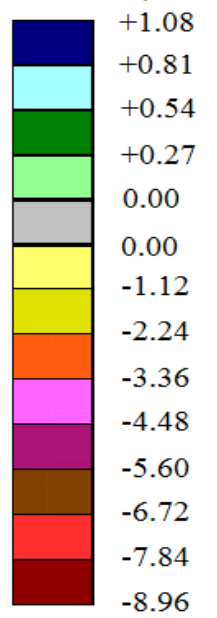

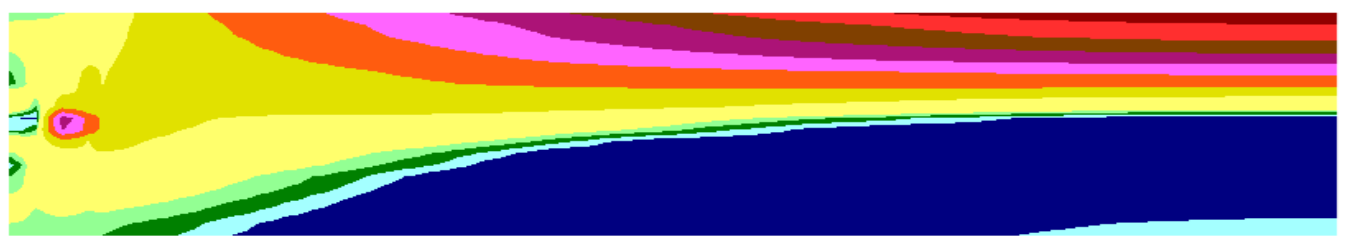

Figura 11. Tensões na direção "x" imediatas ao carregamento para o caso 6 


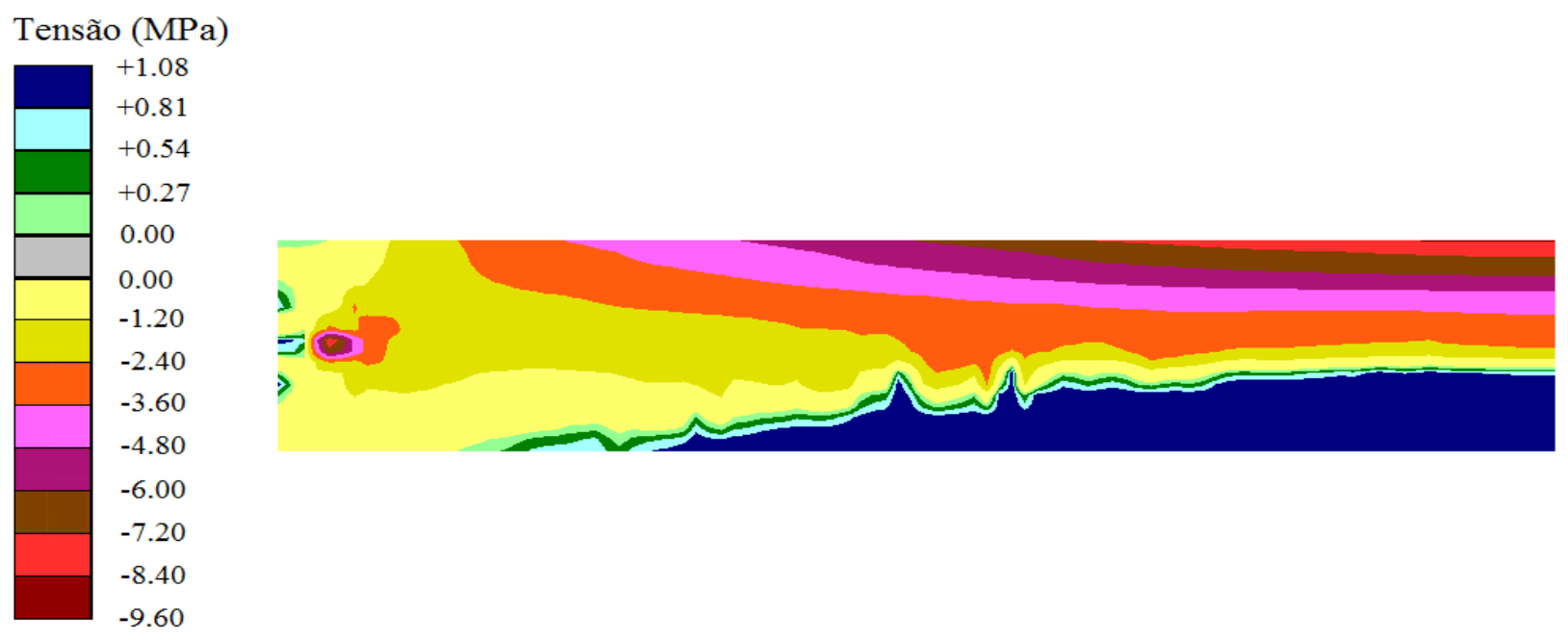

Figura 12. Tensões na direção "x" aos 8000 dias com a RAA para o caso 6

Examinando-se as curvas da Figura 13 observa-se que, para os casos “1”, “4”, “7”, “10”, “13”, "16", "19", "22" e "25", correspondentes às vigas com solicitação externa nula, as tensões nas barras da armadura de aço que no instante imediato ao carregamento apresentavam intensidade de $10 \mathrm{MPa}$, recebeu sobretensão tendo sua intensidade elevada para valor em torno de $30 \mathrm{MPa}$. Para os demais casos o comportamento foi inverso, sendo que para os casos " 3 ", "6", "9", "12", "15", "18", "21", "24" e "27", o alívio de tensões foi de aproximadamente $140 \mathrm{MPa}$ para valor em torno de $110 \mathrm{MPa}$, enquanto para os casos "2", "5", "8", "11", “14", "17”, “20", “23" e "26" constatouse variação de intensidade de $70 \mathrm{MPa}$ para valor em torno de $25 \mathrm{MPa}$. Há, entretanto, de se ponderar que a intensidade das tensões nas barras da armadura de aço no instante imediato ao carregamento assim como suas variações decorrentes do desenvolvimento da RAA não são em nada preocupantes uma vez que as intensidades envolvidas permaneceram bem abaixo da tensões limite de escoamento do material.

As curvas da Figura 14 mostram que, em todos os casos estudados, o efeito expansivo da RAA promoveu a aumento da Tensão Normalizada em compressão. Para os casos "1", "4", “7”, “10”, "13", "16", "19", "22" e "25" o aumento registrado foi discreto perdurando, aos 8000 dias de idade do concreto, margem de segurança considerável. Para os casos "2", "5", "8", "11", "14", "17", "20", "23" e "26" o acréscimo foi de 0,25 para em torno de 0,70 aos 8000 dias de idade do concreto, perdurando uma margem de segurança em torno de 30\%. Em relação aos casos "3", "6", "9", "12", "15", "18”, "21", "24” e "27”, a Tensão Normalizada que ao início das expansões por RAA situavase em torno de 0,45 , apresentou aumento para valor igual a 1,0, determinando uma condição de elevada probabilidade de ruína do concreto. Para os casos "3", "6" e "9", que se referem à temperatura de $20^{\circ} \mathrm{C}$, esta condição seria alcançada aos 3250 dias de idade do concreto. Para os casos " 12 ", " 15 " e " 18 ", concernentes à temperatura de $50^{\circ} \mathrm{C}$, a condição de ruína consumar-se-ia à idade de 2300 dias do concreto, e, para os casos " 21 ", "24" e "27", atinentes a nível térmico de $100^{\circ} \mathrm{C}$, o colapso seria desencadeado aos 1500 dias. Assim, em razão do efeito acelerador exercido pela temperatura sobre a reação álcali-agregado, a ruína do concreto desencadeia-se em idades mais jovens quando o membro estrutural é mantido em ambientes de temperaturas mais elevadas. 


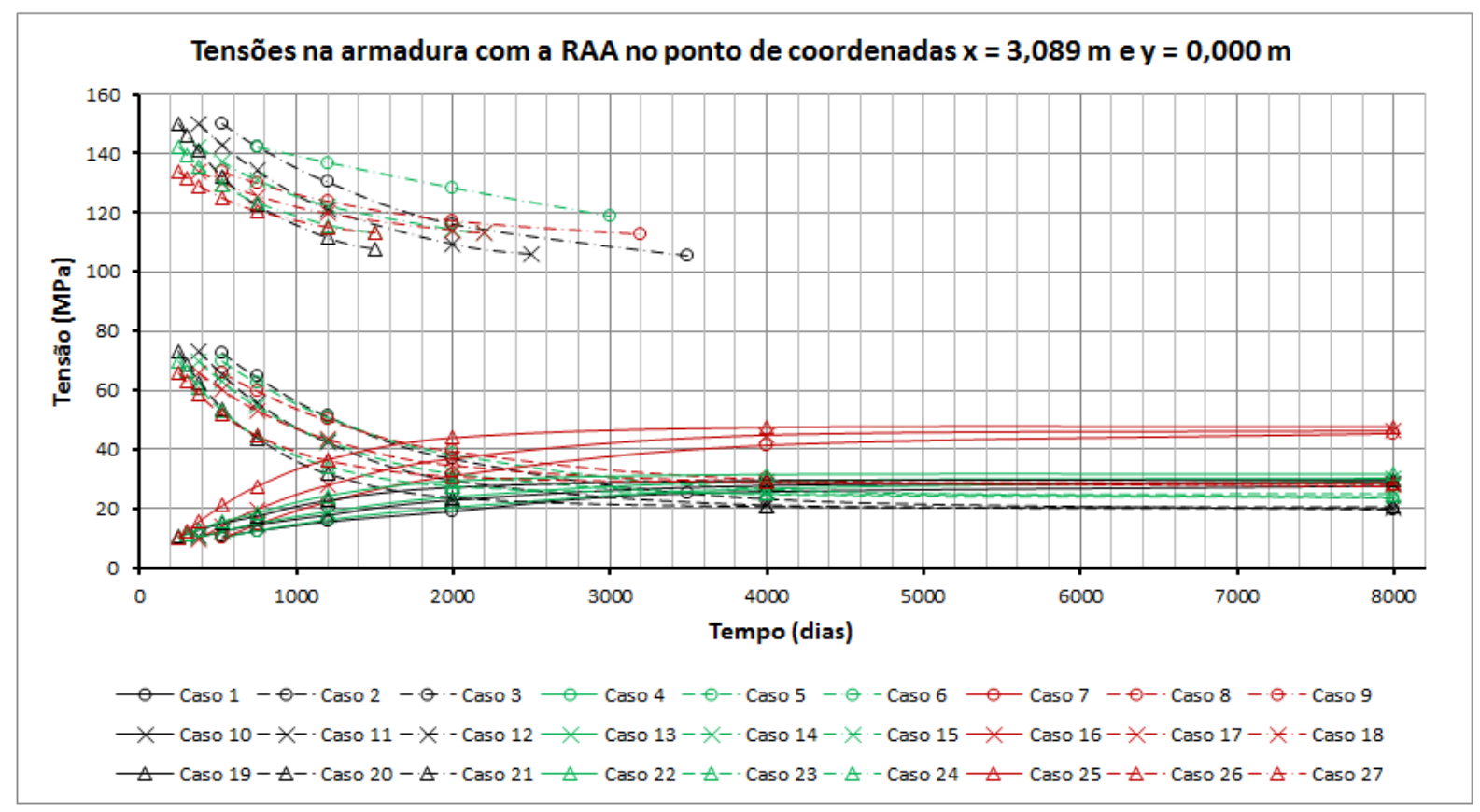

Figura 13. Tensões na armadura na direção "x" devidas à RAA

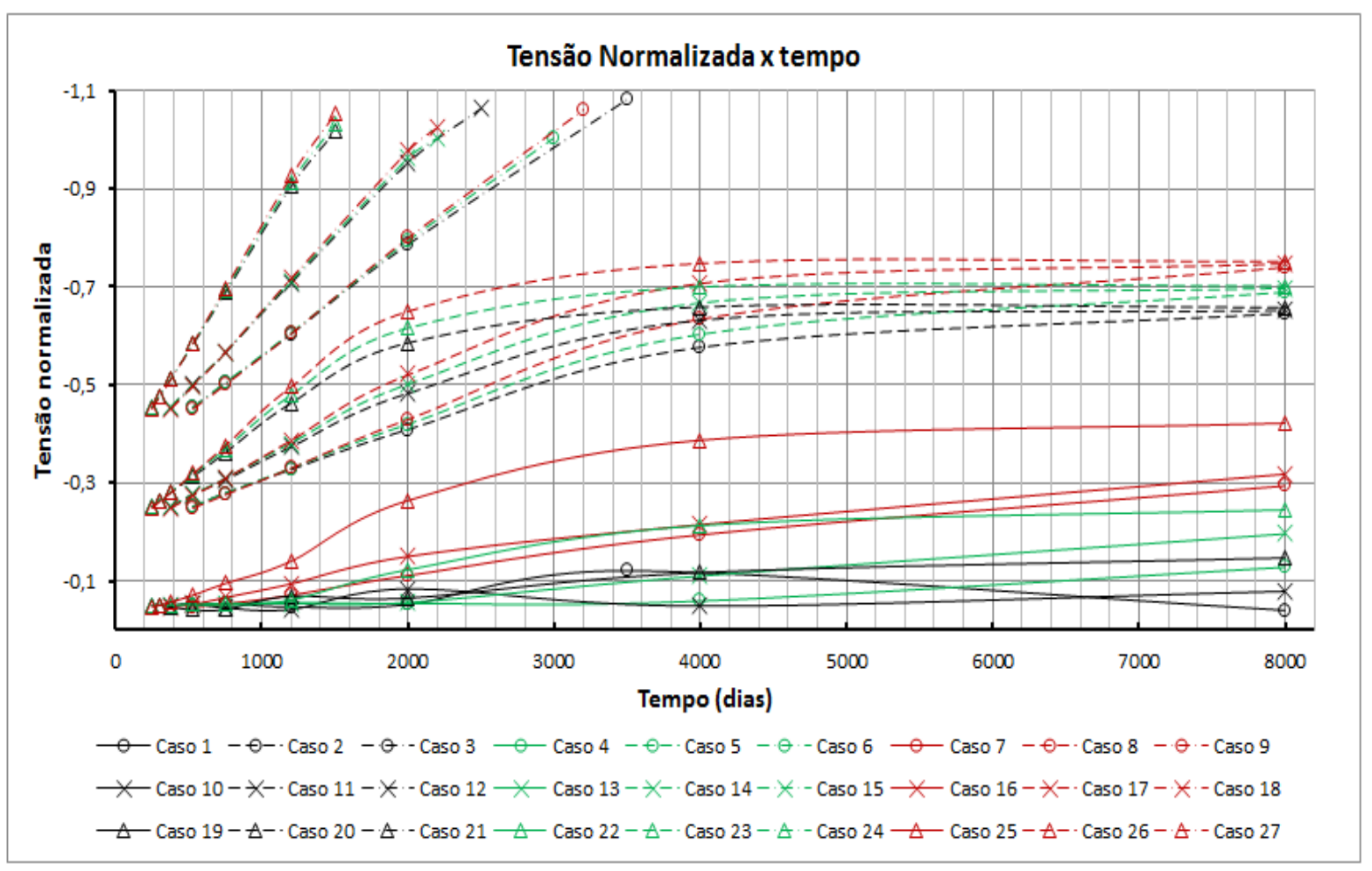

Figura 14. Tensão normalizada com a RAA

Para todos os casos estudados as tensões na massa de concreto na direção "y" apresentaram intensidade pouco significativa à exceção da região da vizinhança dos apoios onde ocorreu perturbação que levou à iminência da ruína. A partir da análise comparativa dos campos ilustrados nessas figuras constata-se a ocorrência de aumento discreto de intensidade de tensões na região da vizinhança dos apoios, bem como a ampliação da zona comprimida da viga como um todo. 
Tabela 3. Resumo de deslocamentos e tensões para todos os casos de análise

\begin{tabular}{|c|c|c|c|c|c|c|}
\hline \multirow[t]{2}{*}{ Caso } & \multicolumn{2}{|c|}{ Deslocamento (mm) } & \multicolumn{2}{|c|}{ Tensão no concreto (MPa) } & \multicolumn{2}{|c|}{$\begin{array}{c}\text { Tensão na Armadura } \\
\text { (MPa) }\end{array}$} \\
\hline & Carregamento & RAA & Carregamento & $\mathbf{R A A}$ & Carregamento & $\mathbf{R A A}$ \\
\hline 1 & $-0,64$ & 2,41 & $-0,99$ & $-0,14$ & 10,42 & 27,74 \\
\hline 2 & $-3,52$ & 1,80 & $-5,00$ & $-3,92$ & 73,59 & 19,54 \\
\hline 3 & $-6,98$ & 1,13 & $-9,01$ & $-8,38$ & 150,66 & 105,51 \\
\hline 4 & $-0,62$ & 2,40 & $-0,97$ & $-0,74$ & 10,07 & 29,38 \\
\hline 5 & $-3,50$ & 1,59 & $-4,98$ & $-4,18$ & 70,50 & 23,74 \\
\hline 6 & $-6,96$ & 0,88 & $-8,98$ & $-8,49$ & 142,70 & 112,99 \\
\hline 7 & $-0,59$ & 2,16 & $-0,94$ & $-1,74$ & 9,53 & 45,42 \\
\hline 8 & $-3,45$ & 1,34 & $-4,94$ & $-4,49$ & 66,53 & 27,71 \\
\hline 9 & $-6,88$ & 0,72 & $-8,95$ & $-8,64$ & 133,97 & 112,68 \\
\hline 10 & $-0,64$ & 2,34 & $-0,99$ & $-0,43$ & 10,42 & 28,78 \\
\hline 11 & $-3,52$ & 1,80 & $-5,00$ & $-3,91$ & 73,59 & 19,94 \\
\hline 12 & $-6,98$ & 1,12 & $-9,01$ & $-8,28$ & 150,66 & 106,01 \\
\hline 13 & $-0,62$ & 2,29 & $-0,97$ & $-1,15$ & 10,07 & 30,27 \\
\hline 14 & $-3,50$ & 1,58 & $-4,98$ & $-4,18$ & 70,50 & 24,12 \\
\hline 15 & $-6,96$ & 0,88 & $-8,98$ & $-8,41$ & 142,70 & 113,07 \\
\hline 16 & $-0,59$ & 2,16 & $-0,94$ & $-1,87$ & 9,53 & 46,38 \\
\hline 17 & $-3,45$ & 1,33 & $-4,94$ & $-4,50$ & 66,53 & 28,08 \\
\hline 18 & $-6,88$ & 0,70 & $-8,95$ & $-8,59$ & 133,97 & 113,22 \\
\hline 19 & $-0,64$ & 2,11 & $-0,99$ & $-0,87$ & 10,42 & 29,63 \\
\hline 20 & $-3,52$ & 1,78 & $-5,00$ & $-3,94$ & 73,59 & 20,62 \\
\hline 21 & $-6,98$ & 1,07 & $-9,01$ & $-8,33$ & 150,66 & 107,79 \\
\hline 22 & $-0,62$ & 2,12 & $-0,97$ & $-1,46$ & 10,07 & 31,68 \\
\hline 23 & $-3,50$ & 1,56 & $-4,98$ & $-4,20$ & 70,50 & 24,91 \\
\hline 24 & $-6,96$ & 0,88 & $-8,98$ & $-8,45$ & 142,70 & 113,06 \\
\hline 25 & $-0,59$ & 1,82 & $-0,94$ & $-2,51$ & 9,53 & 47,69 \\
\hline 26 & $-3,45$ & 1,31 & $-4,94$ & $-4,51$ & 66,53 & 28,79 \\
\hline 27 & $-6,88$ & 0,70 & $-8,95$ & $-8,61$ & 133,97 & 113,30 \\
\hline
\end{tabular}

\section{CONCLUSÕES}

Os resultados obtidos ressaltaram a ação inibidora promovida pela tensão de compressão sobre a expansão por RAA, uma vez que os deslocamentos decorrentes do fenômeno apresentaram magnitudes tanto menores quanto maior a intensidade da referida tensão.

Foi evidenciado, inclusive, o efeito de contenção exercido pelas barras da armadura de aço sobre as deformações que assumiram valores tanto menores quanto maior a taxa de armadura de aço.

Em geral, as expansões por RAA promoveram alívio de tensões de compressão com o tempo na região central do banzo superior da viga, exceto nos casos em que a viga está solicitada apenas por seu peso próprio, exclusivamente, para a maior taxa de armadura, o que pode ser explicado pela 
maior efetividade do efeito de tirante exercido pelas barras de aço de maior área e, portanto, de maior rigidez.

As variações dos campos de tensões com o desenvolvimento da RAA foram discretas, o que deve ser atribuído ao fato de os deslocamentos terem apresentado baixa magnitude, e em razão de a viga ser externamente isostática e o grau de hiperestaticidade interna do conjunto ser insuficiente para provocar redistribuição expressiva de tensões.

A ação deletéria da RAA caracterizou-se em face do empobrecimento do desempenho mecânico promovido pelo fenômeno, uma vez que, apesar da pouca da variabilidade da tensão solicitante, verificou-se aumento das tensões normalizadas em relação à tensão de pico do concreto, ao cúmulo de, especialmente, para os casos de maior tensão inicial, assumir valores iguais à unidade, caracterizando condição iminente de ruína.

Os resultados obtidos revelaram que a influência da temperatura se restringe à cinética da reação, ressaltando que, em temperaturas mais baixas, o fenômeno foi deflagrado, e, posteriormente, estabilizado, em idades mais avançadas, podendo justificar a periodicidade diferenciada para as intervenções corretivas da patologia conforme a temperatura.

\section{REFERENCIAS}

Capra, B. and BournazeL, J. P. (1998), Modelling of Induced Mechanical effects of ÁlkaliAgregate Reactions. Cement and Concrete Research, 28(2):251-260.

Capra, B. and Sellier, A. (2002), Ortotropic Modelling of Alkali-Aggregate Reaction in Concrete Structures: Numerical Simulations. Mechanics of Materials,

Hognestad, E. (1951). A Study of Combined Bending and Axial Load in Reinforced Concrete Members. Bolletin n. 399, Engineering Experiment Station, University of Illinois, Urbana, Illinóis, 49(22).

Kupfer, H.B. and Gerstle, K.H. (1973), Behaviour of Concrete under Biaxial Stresses. Journal of Engineering Mechanics. 99(4):853-866.

Pietruszczac, S. (1996). On the Mechanical Behaviour of Concrete Subjected to Alkali-Aggregate Reaction. Computers \& Structures. 58(6):1093-1097.

Madureira, E.L, Rodrigues, E.C. e da Silva, J.C. (2015), "Simulação Numérica do Efeito da Temperatura em Pilar Parede Submetido à Reação Álcali-Agregado". 57 Congresso Brasileiro de Concreto. Bonito - Mato Grosso do Sul.

RODRIGUES, E.C. (2014), “Análise Numérica do Efeito de Fatores Influentes da Reação ÁlcaliAgregado no Desempenho de Estruturas de Concreto". Dissertação de Mestrado. Programa de PósGraduação em Engenharia Civil. Universidade Federal do Rio Grande do Norte. 